\section{Intravitreal bevacizumab in the treatment of neovascular age- related macular degeneration, 6- and 9-month results}

CA Cleary', S Jungkim², K Ravikumar ${ }^{3}$, C Kelliher' RW Acheson ${ }^{1}$ and M Hickey-Dwyer ${ }^{2}$
${ }^{1}$ Department of Ophthalmology, Mater Misericordiae Hospital, Dublin, Ireland

${ }^{2}$ Department of Ophthalmology, Limerick Regional Hospital, Limerick, Ireland

${ }^{3}$ Department of Ophthalmology, Cork University Hospital, Wilton, Cork, Ireland

Correspondence: CA Cleary, Royal Victoria Eye \& Ear Hospital, Adelaide Road, Dublin 2, Ireland

Tel: + 353872799426 ;

Fax: + 35316761858

E-mail: ucclearly@mac.com

Received: 26 January 2007 Accepted in revised form: 27 April 2007

Published online: 21

September 2007

Part of these data has previously been presented in poster format at the Club Jules Gonin/ Retina Society meeting in Cape Town, South Africa, October 2006

\begin{abstract}
Purpose To evaluate 6- and 9-month follow-up data including the effect on vision and anatomic outcome in patients treated with intravitreal bevacizumab for neovascular age-related macular degeneration (AMD). Study design Interventional consecutive retrospective case series. Patients received intravitreal bevacizumab for the treatment of neovascular AMD including choroidal neovascular membranes, pigment epithelial detachment, and macular haemorrhage. Ophthalmic evaluation included log MAR or Snellen acuity, ophthalmic examination, optical coherence tomography, and fluorescein angiography. Repeat injections were given in the presence of persistent leakage or retinal oedema. Change in vision and foveal thickness from baseline was evaluated using the paired Student's $t$-test.

Results A total of 112 eyes of 111 patients received injections. Median follow-up was 5 months (range: 1-12 months). Mean log MAR vision pre-injection was $0.84 \pm 0.03(n=112)$; at 3 months was $0.69 \pm 0.05(P<0.0001, n=84)$; at 6 months was $0.74 \pm 0.06(P<0.05, n=51)$; and at 9 months was $0.69 \pm 0.08(n=29, P=0.09)$. Thirteen of 17 patients who received only one injection maintained improved or stable vision at 6 months. Mean baseline foveal thickness was $291 \pm 9.72 \mu \mathrm{m}(n=56) ;$ at 3 months was $282.7 \pm 28(P<0.05, n=31)$; and at 6 months was $249.7 \pm 10.3(P<0.05$, $n=12)$. One case of endophthalmitis, three submacular haemorrhages, and three retinal pigment epithelial (RPE) tears occurred.
\end{abstract}

Conclusion Intravitreal bevacizumab is an effective treatment for neovascular AMD, resulting in improved vision and foveal anatomy at 6 months and even up to 9 months. This treatment is well tolerated in the majority of patients but adverse events may include endophthalmitis, RPE tears, and submacular haemorrhage.

Eye (2008) 22, 82-86; doi:10.1038/sj.eye.6702936; published online 21 September 2007

Keywords: age-related macular degeneration (AMD); choroidal neovascularisation (CNV); intravitreal bevacizumab; optical coherence tomography (OCT); retinal pigment epithelial (RPE) tear; submacular haemorrhage.

\section{Introduction}

Vascular endothelial growth factor (VEGF) is known to be the principal mediator of angiogenesis in a variety of ocular conditions including neovascular age-related macular degeneration (AMD). 'Avastin' (Bevacizumab, Genentech, CA, USA) is a recombinant humanised monoclonal IgG1 antibody that binds to and inhibits the biologic activity of human VEGF. Bevacizumab, designed for intravenous administration and first licensed as a treatment for metastatic colon cancer in 2004, is now in widespread use in the treatment of metastatic colon and breast cancer. Systemic bevacizumab was used initially in the treatment of subfoveal choroidal neovascularisation (CNV) in AMD with encouraging results. ${ }^{1}$ The use of intravitreal bevacizumab followed and it 
has been estimated that since then more than 10000 patients have been treated worldwide. ${ }^{2}$ This study describes our experience in the use of intravitreal bevacizumab before the licensing of pegaptanib by the European Agency for the Evaluation of Medicinal Products. At first it was used cautiously in patients who did not fulfill the criteria for photodynamic therapy (PDT) or who were doing poorly on PDT treatment, but as confidence and experience with the drug grew, all patients with classic and occult $\mathrm{CNV}$, serous pigment epithelial detachments (PED), fibrovascular PED, and submacular haemorrhage in AMD were treated.

\section{Patients and methods}

The study design was an interventional consecutive retrospective case series of patients with subfoveal CNV, PED, and submacular haemorrhage who were offered treatment with intravitreal bevacizumab. The off-label use of the drug and its potential risks and benefits were discussed in detail with all patients. A 0.12-ml aliquot of bevacizumab was prepared for each patient and placed in a 1-ml syringe by a compounding pharmacy using aseptic techniques. The eye was prepared using 5\% povidone iodine into the conjunctival fornices and $10 \%$ to the eyelid and periorbital skin: $1.25 \mathrm{mg}$ in $0.05 \mathrm{ml}$ of bevacizumab was injected intravitreally through the pars plana. After the injection, intraocular pressure and retinal artery perfusion were checked and patients were given topical antibiotics to instill four times daily for 1 week. Each patient received a single injection and their progress was monitored at 1 week and then at monthly intervals. Repeat injections were given in the presence of persistent leakage or recurrence of retinal oedema. Clinical evaluation included log MAR or Snellen acuity, complete ophthalmic examination, optical coherence tomography (OCT), ${ }^{3}$ and fluorescein angiography (FA). Ethics Committee approval was obtained for this study.

\section{Statistical analysis}

The main outcome measurements were visual acuity (VA) and central retinal thickness measured by OCT. In 52 patients, Snellen acuities were converted to log MAR for statistical analysis. An increase or decrease in VA was defined as a gain or loss of $\geqslant 5$ letters. The paired Student's $t$-test was used to compare the change in VA and OCT thickness from baseline (Graphpad Prism, Carlsbad, CA, USA). For each paired statistical test, casewise deletion of missing data was carried out. Interval data were analysed for each month up to
6 months. $P<0.05$ was considered to be statistically significant.

\section{Results}

One hundred and twelve eyes of 111 patients were treated. Of the 111 patients who received treatment, 41 were men and 70 were women. Mean patient age was 75 years. Follow-up ranged from 1 to 12 months with a mean and median follow-up of 4.9 and 5 months. The following types of lesion were treated: classic CNV $(n=16)$; occult CNV $(n=22)$; classic with occult CNV $(n=34)$; macular haemorrhage $(n=9)$; and PED $(n=30)$. Of the 30 PEDs, 14 were fibrovascular and 16 were serous. ${ }^{4}$

Mean $\log$ MAR VA pre-injection was $0.84 \pm 0.03$ $(n=112)$. Mean VA at 1 month was $0.67 \pm 0.04(P<0.0001$, $n=100)$; at 2 months was $0.72 \pm 0.04(P<0.0001, n=103)$; at 3 months was $0.69 \pm 0.05(P<0.0001, n=84)$; at 4 months was $0.71 \pm 0.05(P<0.001, n=71)$; at 5 months was $0.70 \pm 0.06(P<0.05, n=54)$; and at 6 months was $0.74 \pm 0.06(P<0.05, n=51)$. This represents a mean gain of $8.5,6,7.5,6.5,7$, and 5 letters from baseline at 1, 2, 3, 4, 5 , and 6 months respectively. Mean VA at 9 months was $0.69 \pm 0.089(n=29, P=0.09)$. Of the 25 patients whose vision improved by greater than 15 letters at 1 month, 14 had a baseline VA 1.0 on the log MAR scale.

Figures 1-3 show the change in VA from baseline at 3,6 , and 9 months.

Table 1 shows the number of letters of VA gained or lost at each month of follow-up.

The mean number of injections per patient was 1.69. Patients most frequently required a second injection at 8

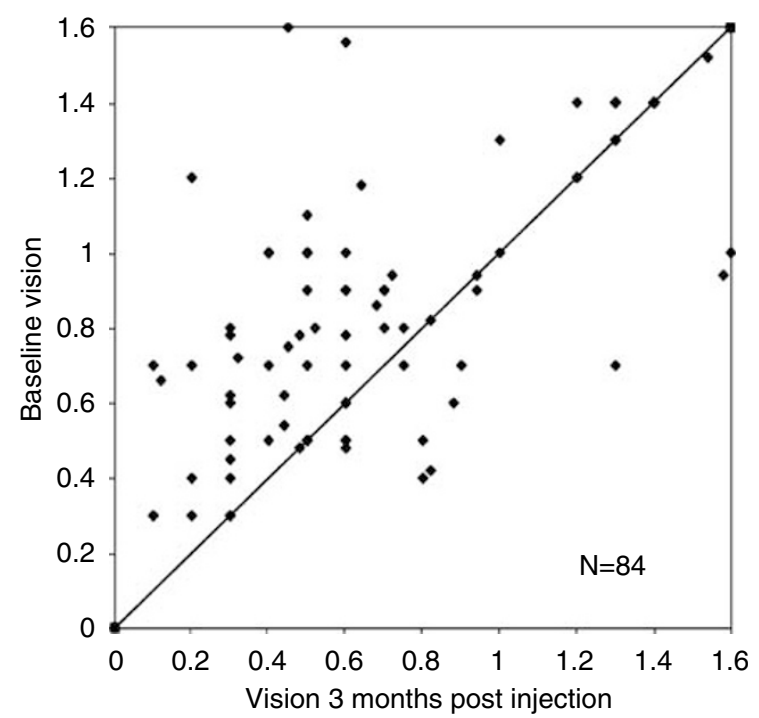

Figure 1 Change in log MAR VA 3 months after intravitreal injection of bevacizumab. 


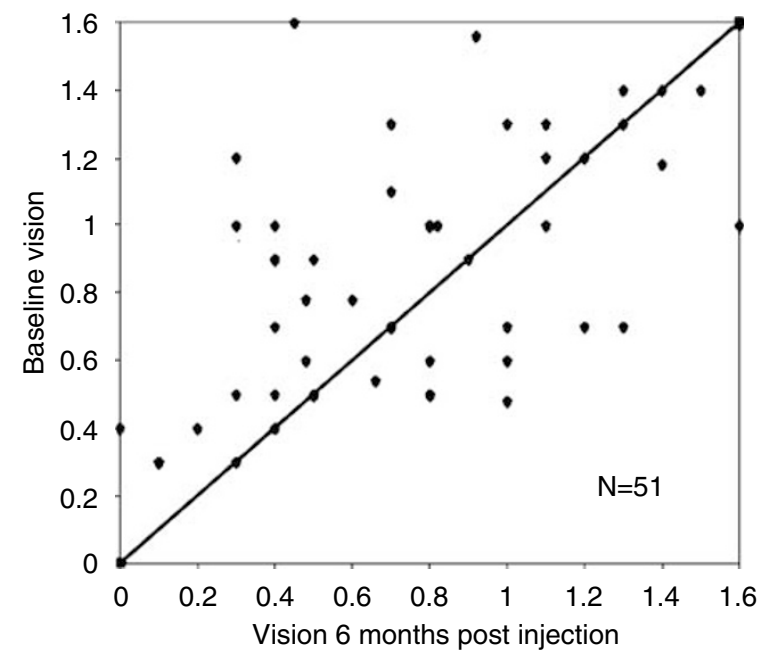

Figure 2 Change in $\log$ MAR VA 6 months after intravitreal injection of bevacizumab.

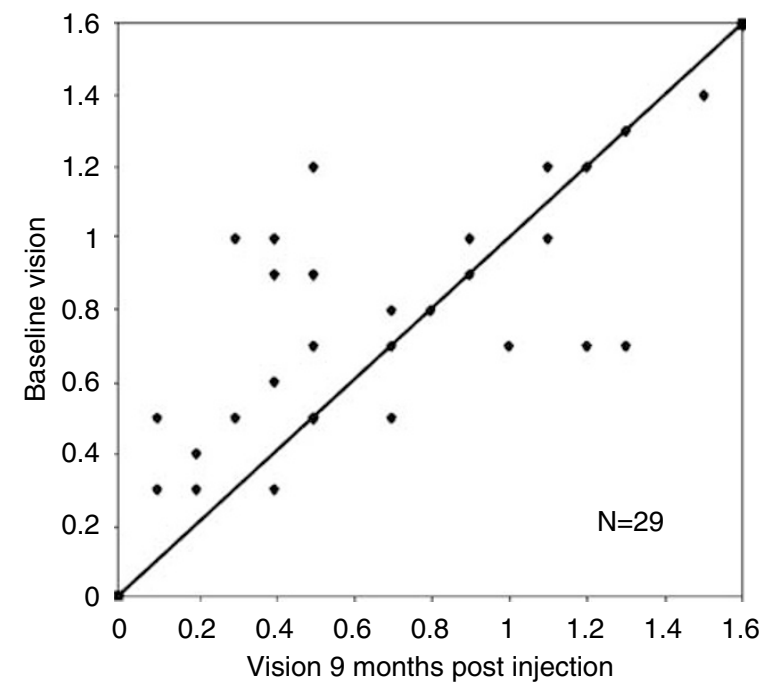

Figure 3 Change in log MAR VA 9 months post-intravitreal injection of bevacizumab.

weeks (mean interval between injections was 11.9 weeks, mode $=8$ weeks). Nineteen patients had previous PDT treatment ${ }^{5}$ of whom one also had intravitreal triamcinolone. No patient had previous anti-VEGF therapy. All but two patients returned for follow-up. Fifty-seven patients received only one injection. Of 17 patients who received only one injection and on whom 6-month follow-up data were available, nine had maintained improved VA, four had stable VA, and four had worse VA. We had one patient with a fibrovascular PED and log MAR VA of 0.45 whose VA improved to 0.18 after only one injection and continued to maintain this level at 11 months follow-up. No patient had raised intraocular pressure, ocular toxicity or uveitis, and no systemic adverse events such as thrombosis or
Table 1 Letters gained or lost at each month of follow-up

\begin{tabular}{lcrrcrrr}
\hline Letters & +15 & +10 & +5 & No change & -5 & -10 & -15 \\
\hline 1 month & 25 & 13 & 13 & 38 & 5 & 2 & 3 \\
$n=99 \%$ & 25 & 13 & 13 & 38 & 5 & 2 & 3 \\
2 months & 30 & 12 & 13 & 30 & 6 & 3 & 9 \\
$n=103 \%$ & 29 & 12 & 13 & 29 & 6 & 3 & 9 \\
3 months & 25 & 10 & 13 & 25 & 3 & 2 & 6 \\
$n=84 \%$ & 30 & 12 & 16 & 30 & 4 & 2 & 7 \\
4 months & 19 & 9 & 11 & 21 & 3 & 2 & 6 \\
$n=71 \%$ & 27 & 13 & 16 & 30 & 4 & 3 & 9 \\
5 months & 16 & 9 & 4 & 16 & 3 & 0 & 6 \\
$n=54 \%$ & 30 & 17 & 7 & 30 & 6 & 0 & 11 \\
6 months & 14 & 7 & 6 & 11 & 3 & 2 & 8 \\
$n=51 \%$ & 28 & 14 & 12 & 22 & 6 & 4 & 16 \\
\hline
\end{tabular}

hypertension were seen. Figure 4 shows a case where improvement in vision and resolution of CNV and PED occurred over an 8-month period.

There were seven ocular adverse events; one case of endophthalmitis, three patients had submacular haemorrhages, and three patients had RPE tears. The first submacular haemorrhage occurred in an 82-year-old man with a PED who developed a large submacular haemorrhage 1 month post-injection with a severe drop in VA. The second submacular haemorrhage occurred in a 56-year-old woman who presented with a right macular haemorrhage and a log MAR VA of 1.0. She had three injections, at baseline, at one, and at 5 months. VA improved to 0.5 at 2 months, and was 0.7 at 5 months. However, a submacular haemorrhage occurred at 6 months with a drop in acuity to 1.1. The third macular haemorrhage occurred in a 77-year-old woman who had a left occult CNV. Baseline log MAR VA was 0.7. She had injections at baseline and at 2 months and VA increased to 0.2 at 3 months. VA dropped to 1.5 at 4 months secondary to a submacular haemorrhage. VA increased slightly to 1.2 at 6 months but there has been no further improvement despite repeated injections at $6,8,10$, and 12 months.

The first patient with an RPE tear was a 63-year-old woman with a classic $\mathrm{CNV}$ and a baseline log MAR VA of 0.6 . VA was 0.6 at 3 months. She developed the RPE tear at 5 months, 1 month after her second injection, and VA dropped to 0.8 . VA had subsequently improved to 0.7 when she was last seen at 8 months. The second patient with an RPE tear was a 77-year-old man who had been treated previously with PDT. Baseline log MAR VA was 0.9 . He had two injections at 1 and 2 months. VA at 3 months was 0.6. He developed an RPE tear at 4 months during which VA was 0.7 . VA subsequently improved in that eye to 0.4 at 6 months and has remained at 0.4 at 9 months. The third patient with an RPE tear was a 68-year-old woman with a PED. Her baseline log MAR VA was 1.1, which improved to 0.5 at 3 months with only 

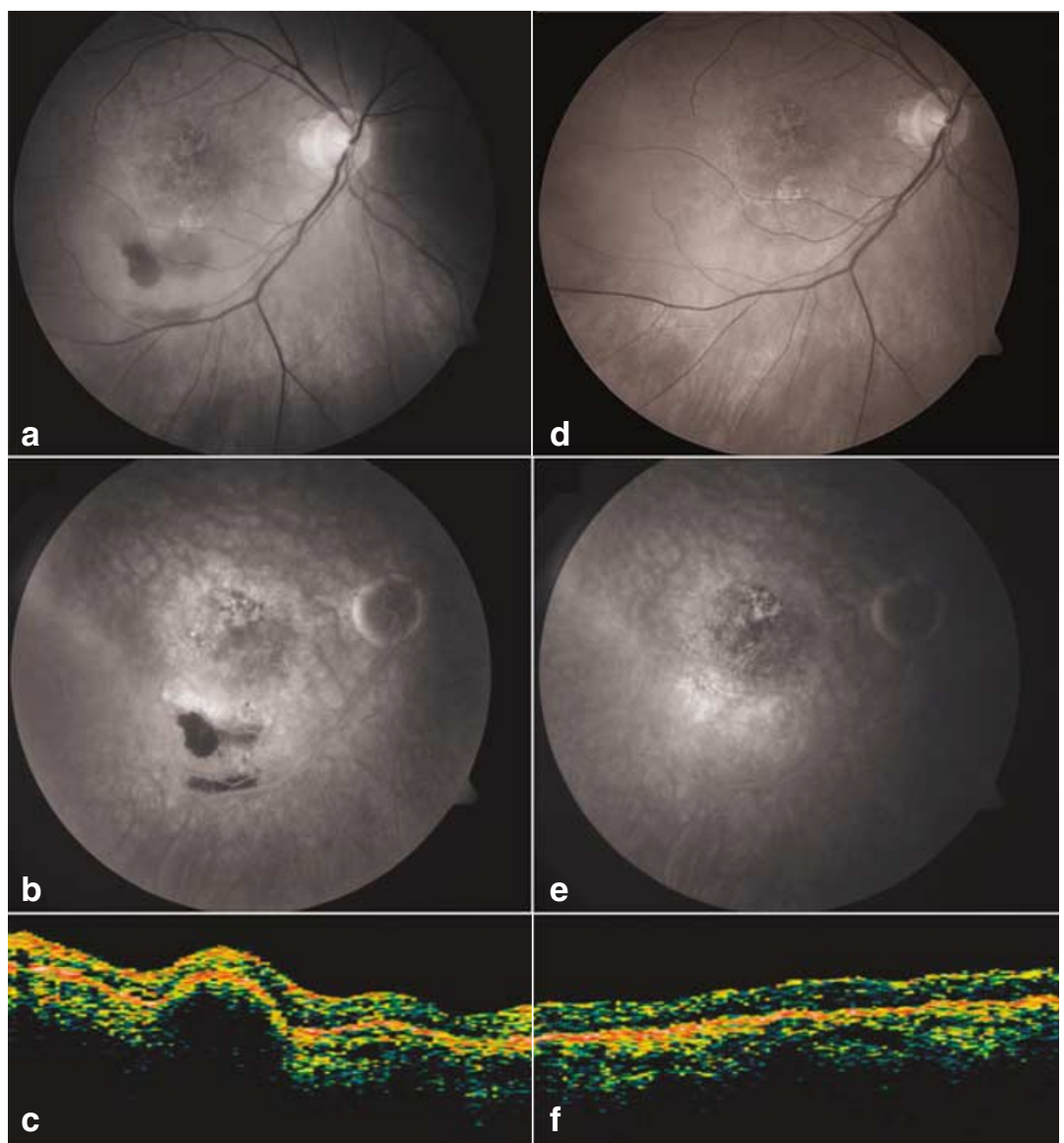

Figure 4 Case report: In 1996, the log MAR VA of this 56-year-old woman was 0.0 in the right eye and 0.4 in the left eye. FA showed an occult CNV at the left macula. In 1997, there was a sudden and permanent drop in VA in the left eye to 1.3 due to a sub-RPE haemorrhage. By 2002 she had developed drusen and occult neovascularisation at the right macula on FA. RVA was 0.2. In 2004, she had a sudden drop in VA to 0.7 in the right eye. A subretinal haemorrhage was seen at the right macula nasal to the fovea. An occult CNV was treated with PDT. In February 2006, VA in the right eye was 0.4. The right macula was flat at the fovea but haemorrhage and PED were present inferior to the fovea (a and b). Foveal thickness was $189 \mu \mathrm{m}$. (c) She received intravitreal bevacizumab in March 2006. By April RVA had improved to 0.2. The macula was flat at the fovea with a reduction in the haemorrhage and PED inferior to the fovea. Foveal thickness was $173 \mu \mathrm{m}$. In June 2006, RVA was 0.2 , the fovea was flat with resolution of the haemorrhage, and the PED was still present inferiorly. Foveal thickness was $162 \mu \mathrm{m}$. In December 2006 ( $\mathrm{d}$ and e), the RVA was maintained at 0.2, the macula was flat (f), the PED had resolved and there was no leak on FA.

one injection. She developed an RPE tear at 6 months post-injection and subsequently a VA of $0.7 . .^{6,7}$

\section{OCT analysis}

One month after injection, resolution of retinal oedema and subretinal fluid was seen on OCT. Mean foveal thickness was $291 \pm 9.72 \mu \mathrm{m}$ at baseline $(n=56)$.

At 1 month, mean foveal thickness was $254.8 \pm 9.74 \mu \mathrm{m}$ $(P<0.0001, n=45)$. At $2,3,4$, and 5 months, mean thickness was $277.2 \pm 18.72(P<0.05, n=32) ; 282.7 \pm 28$ $(P<0.05, n=31) ; 267.2 \pm 19.3(P<0.01, n=20)$; $246.4 \pm 26.9(P=0.28, n=8)$ and at 6 months was $249.7 \pm 10.3(P<0.05, n=12)$. Figure 5 shows the improvement in foveal thickness at 3 months.

\section{Subgroup analysis}

The following subgroups were analysed: classic CNV $(n=16)$; occult CNV ( $n=22)$; classic with occult CNV $(n=34)$; macular haemorrhage $(n=9)$; and PED $(n=30)$. A statistically significant improvement in VA was not achieved within individual subgroups.

\section{Discussion}

This retrospective study demonstrates that intravitreal bevacizumab is an effective treatment for wet AMD, because its use prevented moderate vision loss (as defined by a loss of greater than 15 letters) in $84 \%$ of patients at 6 months. This functional improvement was 


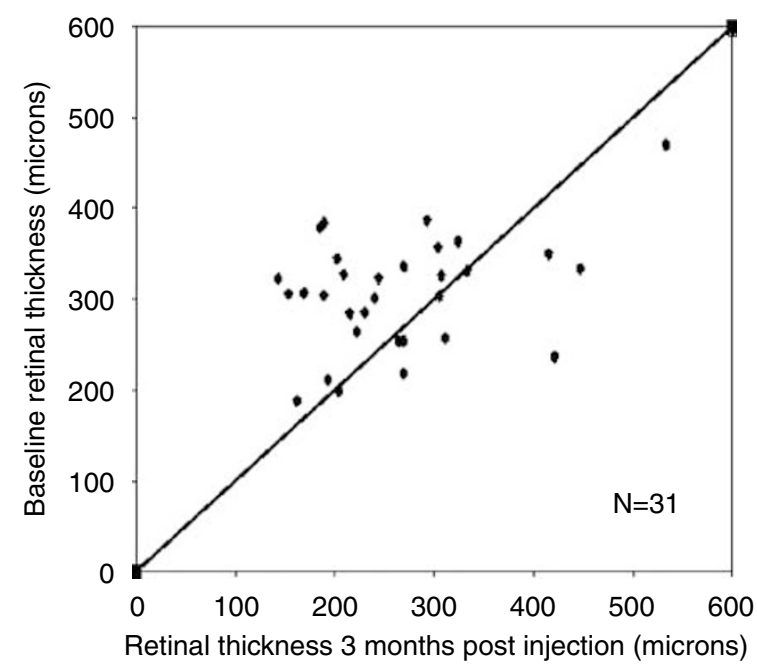

Figure 5 Change in retinal thickness by OCT 3 months after intravitreal injection of bevacizumab.

associated with reduced foveal thickness on OCT. Although similar results have been documented in previous reports at 3 months, ${ }^{8,9}$ here we demonstrate that visual improvement can last 9 months with continued treatment. Indeed, approximately $10 \%$ of patients required only one injection for stabilisation of VA lasting 6 months. Although reinjection appears to be necessary for the majority of patients, the optimum dosing schedule for intravitreal bevacizumab is unknown.

Some patients do not respond. We found worse vision (as defined by a doubling of the visual angle) in between 5 and $10 \%$ of patients after 3 months, which is similar to other reports. ${ }^{9}$ Currently we cannot predict which patients will fail therapy.

For example, we found no difference in response rates between those patients who had received PDT treatment and those who had not. ${ }^{8,9}$ Moreover, even patients with a baseline VA $\leqslant 1.0$ on the log MAR scale had a substantial treatment benefit.

In this study, approximately $15 \%$ (8 of 51) of patients at 6 months lost greater than three lines of vision in spite of treatment. Twenty-five per cent (13 of 51) appeared to improve or stabilise at 6 months with only one injection. The remaining 60\% (30 of 51) required repeated injections for stabilisation of vision. In conclusion, intravitreal bevacizumab appears, in our experience, to be an effective treatment for neovascular AMD.

\section{Acknowledgements}

We are grateful to Dr P Connell, Dr S Charilampidou, Department of Ophthalmology, Mater Misericordiae Hospital, Dublin, Ireland and to Dr D Townley, Department of Ophthalmology, Limerick Regional Hospital, Limerick, Ireland, for their assistance in data collection.

There is no proprietary interest, conflicts of interest, or research funding associated with this work.

\section{References}

1 Moshfeghi AA, Rosenfeld PJ, Puliafito CA, Michels S, Marcus EN, Lenchus JD et al. Systemic bevacizumab (Avastin) therapy for neovascular age-related macular degeneration: twenty-four-week results of an uncontrolled open-label clinical study. Ophthalmology 2006; 113: 2002.e1-2002.e12.

2 Fung AE, Rosenfeld PJ, Reichel E. The International Intravitreal Bevacizumab Safety Survey: using the internet to assess drug safety worldwide. Br J Ophthalmol 2006; 90: 1344-1349.

3 Benson SE, Schlottmann PG, Bunce C, Xing W, Charteris DG. Assessment of the reproducibility and repeatability of a method of grading macular subretinal fluid using optical coherence tomography. Eye 2006; 20: 1030-1033.

4 Casswell AG, Kohen D, Bird AC. Retinal pigment epithelial detachments in the elderly: classification and outcome. Br J Ophthalmol 1985; 69: 397-403.

5 Talks SJ, Setty R, Clarke L. The incidence and outcome of photodynamic therapy for macular degeneration in the Northern region of the UK. Eye 2004; 18: 588-594.

6 Goldstein M, Heilweil G, Barak A, Loewenstein A. Retinal pigment epithelial tear following photodynamic therapy for choroidal neovascularization secondary to AMD. Eye 2005; 19: 1315-1324.

7 Gelisken F, Ziemssen F, Voelker M, Bartz-Schmidt KU. Retinal pigment epithelial tear following intravitreal bevacizumab injection for neovascular age-related macular degeneration. Acta Ophthalmol Scand 2006; 84(6): 833-834.

8 Avery RL, Pieramici DJ, Rabena MD, Castellarin AA, Nasir MA, Giust MJ. Intravitreal bevacizumab (Avastin) for neovascular age-related macular degeneration. Ophthalmology 2006; 113: 363-372. e5.

9 Spaide RF, Laud K, Fine HF, Klancnik JM, Meyerle CB, Yannuzzi LA et al. Intravitreal bevacizumab treatment of choroidal neovascularization secondary to age-related macular degeneration. Retina 2006; 26: 383-390. 